\title{
A magnetic resonance study of complicated early childhood convulsion
}

\author{
R A Grünewald, T Farrow, P Vaughan, C D C Rittey, J Mundy
}

\begin{abstract}
Objectives-The relation between complicated early childhood convulsion (ECC) and adult epilepsy is unclear, although a history of complicated ECC is obtainable in half of adults with epilepsy associated with hippocampal sclerosis. It is not known if the ECC is a marker of preexisting brain damage or is itself harmful to the developing brain. The objective of the study was to assess the extent of structural brain abnormality present soon after a first complicated early childhood convulsion with a view to obtaining data which might contribute to an understanding of whether such abnormalities were likely to be pre-existing or caused by the convulsion.
\end{abstract}

Methods-Children under the age of 5 years were recruited into the study after their first complicated febrile or nonfebrile ECC. None had previously experienced an epileptic seizure. All underwent MRI of the brain within 14 days. Hippocampal volumes and $T 2$ relaxation times were measured. The results were compared with a neurological control group of children without gross structural abnormalities of the neocortex undergoing MRI of the brain for reasons other than epilepsy.

Results-Eighteen patients and 10 control subjects were recruited into the study. One patient was subsequently excluded because of EEG and clinical evidence of benign childhood epilepsy. Nine patients had volumetric evidence of significant hippocampal volume asymmetry (3 SD from the mean of the control group), although in only three of these was the asymmetry apparent on visual inspection of the MRI. Three patients had extrahippocampal neuropathology. None of the control subjects had significant hippocampal volume asymmetry $(p<0.001)$. T2 relaxometry showed no evidence that postictal hippocampal oedema contributed to the asymmetry.

Conclusions-There is a high prevalence of structural brain abnormalities in children within 2 weeks of the first complicated early childhood convulsion, including significant hippocampal asymmetry unrelated to oedema. This does not exclude a damaging effect of complicated ECC on the brain, but suggests that in at least some patients the complicated ECC is the result of pre-existing brain abnormalities.

(F Neurol Neurosurg Psychiatry 2001;71:638-642)
Keywords: early childhood convulsion; hippocampal sclerosis; temporal lobe epilepsy

Early childhood convulsions (ECCs) are epileptic fits in children between the ages of 6 months and 5 years and occur in $2 \%-5 \%$ of the population. ${ }^{1}$ They are probably heterogeneous in aetiology. Hippocampal sclerosis is the commonest single cause of medically refractory temporal lobe epilepsy in adults. ${ }^{2}$ Patients with hippocampal sclerosis, identified either histopathologically after epilepsy surgery, or by MRI, are much more likely than those with other types of localisation related epilepsy to report a history of prolonged or complicated ECC. ${ }^{3-5}$ A complicated ECC is usually defined as a focal seizure, one that is recurrent within 24 hours, one which lasts longer than 20-30 minutes, or one associated with postictal neurological deficit. The severity of the pathology has been reported to be greater the younger the child when the first convulsion occurs. ${ }^{6}$ More severe ECCs are especially associated with the development of localisation related epilepsy and hippocampal sclerosis in later life.

Development of hippocampal sclerosis after ECC does not necessarily imply a cause and effect relation; it may be that pre-existing damage to the hippocampus (for example, hippocampal dysgenesis, anterior or end folium sclerosis, or frank hippocampal sclerosis) predisposes to severe ECC. Many patients with hippocampal sclerosis do not have a history of ECC, ${ }^{8}$ so such convulsions may not be the only cause of hippocampal sclerosis, although they may be one cause. Patients with idiopathic generalised epilepsy or benign childhood partial epilepsy are more likely to have had a febrile convulsion (usually simple) but do not seem to develop hippocampal sclerosis. ${ }^{5}$ Even recurrent ECCs do not necessarily produce hippocampal damage, and it may be the structural brain or metabolic abnormalities present at the time of the ECC which determine whether hippocampal damage ensues. We tested the hypothesis that complicated ECCs may be associated with structural abnormality of the brain, including the hippocampus, which might lead to hippocampal sclerosis and later development of temporal lobe epilepsy.

\section{Methods}

The South Sheffield research ethics committee and all participating District General Hospitals' ethics committees approved the protocol for the study. Parents or guardians of all participating children provided informed consent for inclusion in the study. 
Subjects presenting with first febrile or nonfebrile complicated early childhood convulsion were studied. A complicated ECC was defined as one fulfilling at least one of the following criteria: lasting more than 30 minutes,with focal features (hemiconvulsion, ictal head turning or eye deviation, postictal hemiparesis) or recurrent within 24 hours. Exclusion criteria included a history of previous epileptic seizure, developmental delay, or pre-existing neurological abnormality and contraindication to general anaesthetic. Subjects were referred by paediatricians from the Sheffield Children's Hospital and the surrounding district general hospitals, Doncaster Royal Infirmary, Barnsley District General Hospital, Chesterfield and North Derbyshire Royal Hospital, Rotherham District General Hospital, and The Northern General Hospital, Sheffield. All children underwent MRI of the brain within 2 weeks of their first complicated ECC under general anaesthetic at the University of Sheffield Academic Section of Radiology, Royal Hallamshire Hospital, Sheffield, UK. No definitive power calculation was undertaken as the magnitude of the difference between experimental and control groups was not known before the study was undertaken. A total of 23 children were referred to us for participation in the study. Of these, 18 children aged between 8 months and 5 years were recruited to the study between April 1995 and March 1999. The remainder either had consent refused or could not undergo MRI within 14 days of the convulsion. The clinical condition of the child and the availability of MR scanning time determined the delay in undertaking MRI.

The 10 control subjects were identified from pre-existing MRI records of children between the ages of 6 months and 5 years of age without major cerebrocortical pathology or history of epilepsy. All eligible children were included.

The first 15 MR scans were undertaken on a Siemens Magnetom 63SP, 1.5 Tesla MRI scanner. A coronal MPRAGE T1 weighted scan (TR $11.5 \mathrm{~ms}$, TE $5 \mathrm{~ms}$, TI $300 \mathrm{~ms}$, acquisition time 7 minutes 36 seconds) with coronal $1 \mathrm{~mm}$ thick reconstructions $(0.5 \mathrm{~mm}$ interslice gap), and an axial gradient echo T2 weighted single $4 \mathrm{~mm} 16$ echo CPMG hippocampal T2 relaxometry map perpendicular to the long axis of the hippocampus (TR2200 ms, 16 TEs 22-262 ms, flip angle $90^{\circ}$, acquisition time 8 minutes 38 seconds) were acquired. The last three patients' MR scans were undertaken on a Picker Edge eclipse 1.5T, using a similar protocol except for T2 relaxometry (standard single $4 \mathrm{~mm}$ slice T2 weighted spin echo sequence TR $5000 \mathrm{~ms}$, 4TEs 30, 60, 90, and $120 \mathrm{~ms}$, flip angle $90^{\circ}$, acquisition time $8 \mathrm{~min}-$ utes). MR scans were reported by consultant neuroradiologists and were subsequently analysed volumetrically on a Sparc 20 workstation (SUN Microsystems, CA, USA).

VOLUMETRIC ANALYSIS

One observer (TF) undertook volumetric analysis of the MR scans. Twelve (10 patients, two control subjects) of the $28 \mathrm{MR}$ scans were independently analysed by a second blinded observer (PV; data not shown). Scan data in ACRNEMA (American College of Radiologists. National Electrical Manufacturers Association) format was converted to AnalyzeFormat ${ }^{\circledR}$ within the biomedical imaging package Analyze ${ }^{\circledR}$ version 7.0 (Mayo Clinic, MN, USA). The data sets were reformatted from the posterior horn of the lateral ventricle to the tip of the temporal pole. Reformatted images were resized to cubic voxels of $0.6 \mathrm{~mm}^{3}$.

The hippocampal volume was measured in a posterior to anterior direction. The most posterior slice was identified as the transverse section through the fimbria (where the fornices could be seen projecting beneath the cingulate gyrus, and merging with the hippocampus). All of the grey matter comprising the hippocampus, dentate gyrus, and subiculum were measured, and particular attention paid to excluding the characteristic dark appearing CSF often seen dorsal and lateral to the hippocampus. At the anterior hippocampal head the pes was separated from the amygdala by the often visibly lighter band of cells which form the alveus, or by the visual differences between the hippocampus and amygdala.

For hippocampal measurements a grid of crosses was applied to the coronal images every fifth slice (producing 10 to 12 slices covering the length of the hippocampus). In line with the Cavalieri principle ${ }^{9}$ which enables the calculation of the volume of a structure through random sampling of its cross sectional area, the start slice was randomly selected between zero and the increment minus one from the first slice on which posterior hippocampal tissue was seen. Crosses were removed if the pixel in the innermost part of the chosen corner of the cross comprised hippocampal tissue. For each hippocampal measurement, between 120 and 150 crosses were removed.

A single T2 map was reconstructed from the 16 echo time images of the CPMG sequence from the Siemens scanner ( $22 \mathrm{~ms}$ to $262 \mathrm{~ms}$ ), and from four TEs from the Picker sequence (30, 60, 90, and $120 \mathrm{~ms}$ ). The borders of the coronally visualised hippocampi were manually traced to include as large an area as possible specifically excluding any surrounding CSF. The mean pixel value was recorded (of about 55 pixels).

STATISTICS

All results were analysed with the statistics program SPSS version 7.5.1 using independent samples $t$ tests and $\chi^{2}$ tests. For test-retest repeatability, a coefficient of repeatability was calculated..$^{10}$ This was defined as twice the SD of the mean of the difference between the two raters' hippocampal volume ratio measures, divided by the mean of both raters' hippocampal volume ratio measures of the 12 subjects who had hippocampal volume measurements repeated by the second observer.

\section{Results}

Eighteen subjects who had experienced a complicated ECC and 10 control subjects were recruited to the study. The patient and control groups had a similar sex distribution, but the 
Table 1 Data on experimental and control subjects in study

\begin{tabular}{|c|c|c|c|c|c|c|c|c|c|c|c|c|c|c|}
\hline $\begin{array}{l}\text { No of } \\
\text { subjects }\end{array}$ & $\operatorname{Sex}$ & $\begin{array}{l}\text { Scan age } \\
\text { (days) }\end{array}$ & $\begin{array}{l}R \text { vol } \\
\left(\mathrm{mm}^{3}\right)\end{array}$ & $\begin{array}{l}L \mathrm{vol} \\
\left(\mathrm{mm}^{3}\right)\end{array}$ & $\begin{array}{l}\text { Hippo vol } \\
\text { ratio }\end{array}$ & $\begin{array}{l}R T 2 \\
\text { (ms) }\end{array}$ & $\begin{array}{l}L T 2 \\
(m s)\end{array}$ & $\begin{array}{l}\text { ECC-scan } \\
\text { (days) }\end{array}$ & $\begin{array}{l}\text { ECC Dur } \\
\text { (min) }\end{array}$ & $F H$ & Fever & Focal & Recur & Preg/birth \\
\hline \multicolumn{15}{|c|}{ Patients with ECC: } \\
\hline 1 & $\mathrm{~F}$ & 871 & 2364 & 2378 & 1.01 & 105 & 107 & 9 & 30 & $\mathrm{Y}$ & $\mathrm{Y}$ & HT & $\mathrm{N}$ & $\mathrm{N} /$ Blue \\
\hline 2 & $M$ & 396 & 2039 & 2367 & 1.16 & 115 & 116 & 8 & 45 & $\boldsymbol{N}$ & $\boldsymbol{Y}$ & $\boldsymbol{L}$ & $N$ & N/Pro \\
\hline 3 & $M$ & 1662 & 2880 & 2635 & 1.09 & 109 & 105 & 5 & 30 & $\boldsymbol{Y}$ & $N$ & $\boldsymbol{G}$ & $N$ & $N / N$ \\
\hline 4 & $\mathrm{~F}$ & 311 & 1935 & 1935 & 1.00 & 124 & 120 & 13 & 35 & $\mathrm{Y}$ & $\mathrm{Y}$ & G & $\mathrm{N}$ & N / Pro \\
\hline 5 & $\mathbf{M}$ & 384 & 2903 & 2160 & 1.34 & 123 & 119 & 9 & 150 & $\mathbf{Y}$ & $\mathbf{Y}$ & G & $\mathbf{N}$ & N / Pro \\
\hline 6 & M & 269 & 2074 & 2157 & 1.04 & 127 & 125 & 13 & 5 & $\mathrm{~N}$ & $\mathrm{Y}$ & $\mathrm{R}$ & $\mathrm{N}$ & $\mathrm{N} / \mathrm{Csn}$ \\
\hline 7 & $\mathbf{F}$ & 508 & 2817 & 3525 & 1.25 & 121 & 111 & 13 & 40 & $\mathbf{N}$ & $\mathbf{Y}$ & G & $\mathbf{N}$ & N/Csn \\
\hline 8 & $\mathbf{F}$ & 503 & 2722 & 2239 & 1.22 & 107 & 112 & 10 & 20 & $\mathbf{N}$ & $\mathbf{Y}$ & $\mathbf{L}$ & $\mathbf{N}$ & Bld / N \\
\hline 9 & $\mathrm{M}$ & 520 & 2091 & 1970 & 1.06 & 124 & 124 & 7 & 135 & $\mathrm{Y}$ & $\mathrm{Y}$ & G & $\mathrm{Y}$ & Bld / Csn \\
\hline 10 & $\mathbf{M}$ & 354 & 2474 & 2848 & 1.15 & 126 & 121 & 11 & 2 & $\mathbf{N}$ & $\mathbf{Y}$ & G & $\mathbf{Y}$ & $\mathbf{N} / \mathbf{N}$ \\
\hline 11 & M & 531 & 2748 & 2661 & 1.03 & 123 & 121 & 14 & 180 & $\mathrm{Y}$ & $\mathrm{N}$ & G & $\mathrm{N}$ & $\mathrm{N} /$ Ind \\
\hline 12 & $\mathbf{M}$ & 518 & 2903 & 2129 & 1.36 & 114 & 114 & 14 & 40 & $\mathbf{Y}$ & $\mathbf{Y}$ & $\mathbf{R}$ & $\mathbf{Y}$ & Ind $/ \mathbf{N}$ \\
\hline 13 & $\mathbf{F}$ & 670 & 2575 & 2367 & 1.09 & 141 & 129 & 14 & 30 & $\mathbf{Y}$ & $\mathbf{Y}$ & $\mathbf{R}$ & $\mathbf{Y}$ & N / Csn \\
\hline 14 & M & 723 & 2402 & 2350 & 1.02 & 112 & 103 & 5 & 20 & $\mathrm{~N}$ & $\mathrm{~N}$ & $\mathrm{R}$ & $\mathrm{N}$ & $\mathrm{N} / \mathrm{N}$ \\
\hline 15 & $\mathbf{M}$ & 386 & 2170 & 1922 & 1.13 & 125 & 121 & 14 & 30 & $\mathbf{N}$ & $\mathbf{Y}$ & G & $\mathbf{N}$ & $\mathbf{N} / \mathbf{N}$ \\
\hline 16 & $\mathrm{~F}$ & 764 & 1827 & 1866 & 1.02 & 123 & 115 & 13 & 30 & $\mathrm{~N}$ & $\mathrm{~N}$ & G & $\mathrm{N}$ & $\mathrm{N} / \mathrm{N}$ \\
\hline 17 & M & 409 & 2091 & 2160 & 1.03 & 135 & 130 & 12 & 45 & $\mathrm{Y}$ & $\mathrm{N}$ & G & $\mathrm{N}$ & $\mathrm{N} / \mathrm{N}$ \\
\hline \multirow[t]{3}{*}{18} & $\mathbf{M}$ & 811 & 1866 & 1617 & 1.15 & 135 & 122 & 4 & 90 & $\mathbf{N}$ & $\mathbf{Y}$ & G & $\mathbf{N}$ & $\mathbf{N} / \mathbf{N}$ \\
\hline & $\begin{array}{l}\text { Mean } \\
\text { (SD) }\end{array}$ & $\begin{array}{l}525 \\
(182)\end{array}$ & $\begin{array}{l}2353 \\
(372)\end{array}$ & $\begin{array}{l}2274 \\
(438)\end{array}$ & $\begin{array}{l}1.12 \\
(0.11)\end{array}$ & $\begin{array}{l}122 \\
(9)\end{array}$ & $\begin{array}{l}118 \\
(7)\end{array}$ & $\begin{array}{l}11 \\
(3)\end{array}$ & $\begin{array}{l}53 \\
(51)\end{array}$ & & & & & \\
\hline & Sex & $\begin{array}{l}\text { Scan age } \\
\text { (days) }\end{array}$ & $\begin{array}{l}R \text { vol } \\
\left(\mathrm{mm}^{3}\right)\end{array}$ & $\begin{array}{l}L \text { vol } \\
\left(\mathrm{mm}^{3}\right)\end{array}$ & $\begin{array}{l}\text { Hippo vol } \\
\text { ratio }\end{array}$ & \multicolumn{9}{|c|}{ Reason for MRI } \\
\hline \multicolumn{15}{|c|}{ Control subjects: } \\
\hline 19 & $\mathrm{~F}$ & 970 & 2986 & 3069 & 1.03 & \multicolumn{9}{|c|}{ Occulomotor nerve angioma/neurofibroma } \\
\hline 20 & $M$ & 461 & 1440 & 1373 & 1.05 & \multicolumn{9}{|c|}{ Cleft palate } \\
\hline 21 & M & 1560 & 1360 & 1350 & 1.01 & \multicolumn{9}{|c|}{ Dysarthria, spastic dysplegia } \\
\hline 22 & $\mathrm{M}$ & 607 & 3093 & 2989 & 1.03 & \multicolumn{9}{|c|}{ Hypotonia, poor visual development } \\
\hline 23 & M & 549 & 1852 & 1839 & 1.01 & \multicolumn{9}{|c|}{ Hypotonia, visual impairment, cortical myoclonus } \\
\hline 24 & $\mathrm{M}$ & 1383 & 3380 & 3338 & 1.01 & \multicolumn{9}{|c|}{ Idiopathic growth hormone deficiency } \\
\hline 25 & $\mathrm{~F}$ & 332 & 1167 & 1145 & 1.02 & \multicolumn{9}{|c|}{ Pituitary mass } \\
\hline 26 & $\mathrm{~F}$ & 1380 & 1720 & 1802 & 1.04 & \multicolumn{9}{|c|}{ Craniopharyngioma } \\
\hline 27 & $\mathrm{~F}$ & 366 & 2876 & 2944 & 1.02 & \multicolumn{9}{|c|}{ Abnormality of pituitary stalk/hypothalamus } \\
\hline \multirow[t]{3}{*}{28} & M & 564 & 2227 & 2400 & 1.07 & \multirow{3}{*}{\multicolumn{9}{|c|}{ Dysmorphic features with developmental delay }} \\
\hline & Mean & 817 & 2210 & 2225 & 1.03 & & & & & & & & & \\
\hline & $(\mathrm{SD})$ & $(442)$ & $(772)$ & $(779)$ & $(0.02)$ & & & & & & & & & \\
\hline
\end{tabular}

Subjects with hippocampal ratios $\geqslant 1.09$ are shown in bold typeface; ECC scan=time in days between convulsion and MRI scan; ECC dur=duration of convulsion; $\mathrm{FH}=$ family history of epilepsy; Fever=presence of fever on arrival at hospital; Focal =any focal features $(\mathrm{R}, \mathrm{L}=$ right or left hemiconvulsion) or whether generalised (G); Recur=recurrence of seizures in 24 hour period; $H T=$ head turning; $N=$ normal; $C s n=$ caesarian section; Pro=prolonged labour; Bld=vaginal bleeding during pregnancy; Ind=induced labour; Patient 3 (in italics) was excluded because of clinical and EEG evidence of benign childhood epilepsy, and the data were not included in means and SD calculations for the "patients with ECC" group).

control group had a slightly older age at scan (mean (SD) 817 (442) days versus 525 (182) days $(\mathrm{p}=0.025)$ by independent samples $t$ test, table 1). One of the 18 patients was excluded from the study because of the history and EEG evidence of benign childhood epilepsy with centrotemporal spikes.

Limited clinical information was available from those who were referred for study but were not studied because their guardians refused consent or MRI could not be undertaken within 14 days of ECC. There was no difference in age between the participating and non-participating children (mean age at ECC of participants 566 (SD 327) days, nonparticipants 588 (SD 309) days, $\mathrm{p}=0.9$ by independent samples $t$ test).

Four of the $17 \mathrm{MR}$ scans of the group that had experienced ECC were reported as abnormal by the neuroradiologists. Hippocampal volumes and volume ratios are shown in table 1. Three of the nine patients with significant hippocampal volume asymmetry (defined as equal to $3 \mathrm{SD}$ from the mean of the control group (1.09) were reported by the radiologist as having hippocampal asymmetry. One patient with symmetric hippocampi (volume ratio 1.04) was reported as having significant asymmetry by our neuroradiologists.

The coefficient of repeatability for the hippocampal volume ratios of the 12 children whose MR scans were assessed by the second observer (PV) was $14 \%$, implying good agreement between the observers.

Three patients $(2,12$, and 15 , all with hippocampal volume ratios $\geqslant 1.09$ ) had radiologically reported extrahippocampal neuropathology. These comprised a mature (perinatal or prenatal) infarct in the right middle cerebral artery territory, left temporal polymicrogyria, and left temporal lobe heterotopia, respectively.

Although none of the 10 control subjects had a hippocampal volume ratio greater than 1.07 , in nine of the 17 patients hippocampal ratios were equal to 1.09 (mean 1.21 (0.09), range 1.09 to $1.36, \mathrm{p}<0.001$ by $\chi^{2}$ test).

Mean hippocampal volumes were similar in children with ECC and control subjects. There was no MRI evidence of hippocampal dysgenesis, end folium sclerosis, or frank hippocampal sclerosis in any subject. However, there was a significantly greater volume asymmetry in the group who had experienced ECC than the control group (ECC group mean difference between larger and smaller hippocampus 271 (SD 271) $\mathrm{mm}^{3}$, control group 66 (SD 49) $\mathrm{mm}^{3}$, $\mathrm{p}=0.028$ by independent samples $t$ test). The hippocampal volume ratio (expressed as larger/ smaller hippocampus) was significantly greater in the ECC group compared with the control group (experimental group 1.12 (SD 0.11), control group 1.03 (SD 0.02), $\mathrm{p}=0.009$ by independent samples $t$ test). No control subject had a hippocampal volume ratio >1.07. 
Subjects with hippocampal asymmetry (volume ratios $\geqslant 1.09$ ) had similar seizure duration to those with symmetric hippocampi (48 (SD 43) minutes. $v 60$ (SD 58) minutes, $\mathrm{p}=0.65$ by independent samples $t$ test), and a similar prevalence of pregnancy and birth complications.

Eight of the 17 patients with ECC had a family history of epilepsy and 13 were febrile on admission to hospital. Six had focal features to their convulsion, 10 had a prolonged generalised seizure, and one had ictal head turning to the left. Three had convulsions lasting less than 30 minutes but with focal features (three with Todd's paresis). In four patients the seizure recurred within 24 hours. Fourteen of the 17 mothers in the experimental group had a normal pregnancy, the remaining three having minor vaginal bleeding or placental failure. Six patients had normal gestation and births, two were born by caesarian section, three were induced (one of these for placental failure), one was a face presentation, and three had a prolonged second stage of labour.

Of the nine patients with a hippocampal ratio $\geqslant 1.09$, four had focal features, of which three had a larger hippocampal volume on the same side as the clinical seizure focus and one a larger volume on the opposite side. All nine had febrile seizures but only three had a family history of epilepsy. The 13 with febrile seizures had significantly greater volume asymmetry than the four with afebrile seizures (mean hippocampal ratio for those with febrile seizures 1.15 (SD 0.12), afebrile seizures 1.03 (SD $0.006), \mathrm{p}<0.05)$.

T2 relaxometry was undertaken in all 17 of the patients who had experienced ECC. There was no significant difference in hippocampal T2 relaxation time between left and right hippocampi in the experimental group, larger and smaller hippocampi in the whole group, or between larger and smaller hippocampi in the subgroup of patients with hippocampal volume ratios $=1.09$. Nor was there any difference between hippocampal T2 relaxation times ipsilateral or contralateral to the presumed seizure focus in the children with a focal component to their seizures.

\section{Discussion}

The results of the study can be summarised as follows:

(1) There was a high prevalence of abnormalities of the brain in patients undergoing MRI within 2 weeks of their first complicated ECC (nine of 17 patients, 53\%).

(2) Patients who had experienced a complicated ECC often had significant hippocampal volume asymmetry whereas no such asymmmetry was found in control subjects.

(3) There was no apparent relation between pregnancy or birth complications, age at first complicated ECC, features of the epileptic seizure, or family history and the presence of significant hippocampal volume asymmetry in the group which had experienced ECC.

(4) Hippocampal T2 relaxometry measurements were similar in larger and smaller hippocampi to those with hippocampal volume asymmetry, implying that at a median of 11 days from seizure to MR investigation, such asymmetry could not, as suggested by other authors, ${ }^{11}$ be ascribed to hippocampal oedema.

The relation between hippocampal damage and ECC is complex. Previous studies of the relation between ECC and later epilepsy have suggested that the associated risk of epilepsy is small. ${ }^{1}$ It is considerably greater if the child experiences a prolonged seizure, a seizure of focal onset, has postictal lateralised weakness (Todd's paresis), or has recurrent seizures within 24 hours. ${ }^{12}{ }^{13}$ We therefore concentrated on this group to determine if any abnormalities were detectable on MRI of the brain as soon after the convulsion as we could undertake the examination. As hippocampal abnormalities were already present within 2 weeks of a first complicated ECC, it is possible that hippocampal damage antedated first complicated ECC rather than resulted from it. Some abnormalities detected in such a study may have developed in the days between the convulsion and the MRI. However, it has been recently shown that family members of children with hippocampal sclerosis had a high prevalence of significant hippocampal asymmetry, even if asymptomatic, and it may be that we have found a similar hippocampal abnormality which can progress to hippocampal sclerosis in certain circumstances. ${ }^{14}$ An alternative explanation is that sufficient hippocampal damage had already taken place by the time of the MRI to cause unilateral hippocampal atrophy but without gliosis sufficient to increase T2 relaxation time.

The study showed a high prevalence of abnormalities on MRI of the brain within 2 weeks of first complicated ECC. Four of the 17 children had abnormalities identified by neuroradiologists, but one proved spurious on measurement of the hippocampal volumes. A further six patients had abnormal hippocampal asymmetry on volumetric analysis which was not initially detected by neuroradiologists. This reinforces published findings that visual inspection of hippocampi on MRI can be misleading. ${ }^{15}$

Hippocampal T2 relaxometry has been used to help identify hippocampal sclerosis in adults. ${ }^{515}$ In our experience hippocampal T2 relaxation time is variable in early childhood and it has not been used as a diagnostic tool to detect hippocampal sclerosis. However, T2 relaxometry is a sensitive indicator of oedema. ${ }^{16}$ There was no consistent asymmetry of T2 relaxation time in our study group, even in those with focal features to their seizure or significant hippocampal asymmetry. This suggests that hippocampal oedema is unlikely to contribute significantly to the hippocampal asymmetry found.

Although it is now well established that hippocampal volumes are symmetric (usually to within $5 \%$ ) in the normal adult population, the range of hippocampal asymmetry has not yet been demonstrated in normal children. We therefore included a neurological control group of 10 children who were undergoing MRI of the brain for indications other than 
epilepsy and who did not have major structural abnormalities of the temporal lobe. Despite the presence of significant neurological disease in this group, hippocampal volumes were symmetric to within $7 \%$. We adopted a stringent definition of hippocampal asymmetry as equal to $3 \mathrm{SD}$ from the mean of this control group.

Subgroup analysis showed that the patients whose complicated ECC was associated with fever had significantly greater hippocampal asymmetry than those whose ECC was afebrile. Although the value of such an analysis is limited, this is consistent with the finding that seizures associated with fever are more likely to damage the hippocampus in experimental animals. ${ }^{17}$

In a previous study, three of the 20 patients who had complicated ECC had a clear history of unilateral febrile seizures, and all three had small hippocampal volumes on the side contralateral to the spasms. ${ }^{8}$ Non-febrile ECCs were not associated with hippocampal volume loss. These authors suggested that it is possible that fever in itself has an influence or that febrile seizures occurred earlier in life or were more prolonged. In this study, hippocampal volume asymmetry was not particularly associated with focal ECC, nor was the smaller hippocampus consistently contralateral to the presumed seizure focus. It is therefore likely that hippocampal pathology progresses in at least some patients after the first complicated ECC. This has been investigated by VanLandingham et al, who performed MRI on 27 infants soon after complicated ECC. ${ }^{11}$ In several patients whose initial MRI was abnormal, a follow up study was performed 8 to 10 months after the initial ECC to determine if permanent injury had occurred in these patients. Initial MRI took place within 6 days of the seizure in all but one patient, but importantly these were not necessarily the children's first seizure. Definite MRI abnormalities were seen in six of the 15 infants with focal or lateralised ECCs, but none were seen in the 12 infants with generalised ECCs. Two of the patients with lateralised seizures had bilateral hippocampal atrophy, suggesting a history of perinatal injury. The remaining four patients had increased hippocampal volumes on the side of seizure origin. This was attributed to oedema, as visual inspection of the MRI suggested increased hippocampal T2 signal. T2 relaxometry was not performed. Two of these four patients with increased hippocampal volumes were rescanned after 8 to 10 months and both then showed hippocampal atrophy. One of these patients was rescanned again at 25 months during which time the affected hippocampus had grown, but failed to reach the normal volume range. Their interpretation of this sequence of scan findings was that the hippocampi were initially normal, then injured by the seizures and consequently developed atrophy. Our data, by contrast, suggest that hippocampi in a high proportion of such patients are initially abnormal, but our data relate to an earlier stage in the progression of hippocampal pathology. We agree, however, with their conclusion that some complicated ECCs may be related to pre-existing brain dysfunction and that ECCs themselves may produce a further insult to the hippocampus that may evolve to hippocampal atrophy.

The study was supported by the Children's Hospital Research in Sheffield (CHRIS) fund, a registered UK charity. We thank the neuroradiologists of the Royal Hallamshire Hospital (Professor P Griffiths, Dr U Bergvall, Dr T Powell, Dr T Hodgson) for P Griffiths, Dr U Bergvall, Dr T Powe
their help in interpreting the MR scans.

RAG and CDCR designed the study, which was undertaken RAG and CDCR designed the study, which was undertaken
by RAG, TF, and JM. Data were analysed by RAG, TF, and PV. by RAG, TF, and JM. Data were analysed by RAG, TF, and PV.
RAG and TF wrote the manuscript with contributions from all RAG and TF
other authors.

1 Verity CM, Golding J. Risk of epilepsy after febrile convulsions: a national cohort study. BM尹 1991;303:1373-6.

2 Babb TL, Brown WJ. Pathological findings in epilepsy. In: J Engel Jr, ed. Surgical treatment of the epilepsies. New York: Raven Press, 1987:520-4.

3 DeGiorgio CM, Tomiyasu U, Gott PS, et al. Hippocampal pyramidal cell loss in human status epilepticus. Epilepsia 1992;33:23-7

4 Dam AM. Epilepsy and neuron loss in the hippocampus. Epilepsia 1980;21:617-29.

5 Grünewald RA, Jackson GD, Connelly A, et al. MR detection of hippocampal pathology in epilepsy: factors influencing T2 relaxation time, AfNR Am $\mathcal{F}$ Neuroradiol 1994,15:1149-56

6 Sagar HJ, Oxbury JM. Hippocampal neuron loss in temporal lobe epilepsy: correlation with early childhood convulsions. Ann Neurol 1987;22:334-40.

7 Wallace SJ. Spontaneous fits after convulsions with fever. Arch Dis Child 1977,52:192-6.

8 Kuks JBM, Cook MJ, Fish DR, et al. Hippocampal sclerosis in epilepsy and childhood febrile seizures. Lancet 1993;342: in epileps.

9 Cavalieri B. Geometrica indivisibilibus continurium (typis clementis ferronij, bononiae), 1635. Reprinted as: Geometrica degli indivisibili. Torino: Unione Tipografico-Editrice Torinese, 1966

10 Bland JM, Altman DG. Statistical methods for assessing agreement between two methods of clinical measurement. Lancet 1986;i:307-10.

11 VanLandingham KE, Heinz ER, Cavazos JE, et al. Magnetic resonance imaging evidence of hippocampal injury after prolonged focal febrile convulsions. Ann Neurol 1998;43: prolong 26 .

12 Wallace SJ. Prognosis after prolonged unilateral febrile convulsions. In: Akimoto $\mathrm{H}$, Kazamatsur $\mathrm{H}$, Seino $\mathrm{M}$, eds. Advances in Epileptology XIIIth Epilepsy International Symposium. New York: Raven, 1992:97-9.

13 Awaya Y, Iwamoto H, Fukuyama Y. A long-term follow-up study of first episodes of idiopathic status convulsivus in childhood in relation to subsequent epilepsy. fapanese fourchildhood in relation to subsequent epilepsy.
nal of Psychitric Neurology 1992;46:303-6.

14 Fernández G, Effenberger O, Vinz B, et al. Hippocampal malformation as a cause of familial febrile convulsions and subsequent hippocampal sclerosis. Neurology 1998;50:90916

15 Jackson GD, Connelly A, Duncan JS, et al. Detection of hippocampal pathology in intractable partial epilepsy: increased sensitivity with quantitative magnetic resonance T2 relaxometry. Neurology 1993;43:1793-9.

16 Barnes D, McDonald W, Tofts P, et al. Nuclear magnetic resonance imaging in experimental cerebral oedema. $\mathcal{F}$ Neurol Neurosurg Psychiatry 1986;49:1341-7.

17 Meldrum BS, Brierley J B. Prolonged epileptic seizures in primates: ischaemic cell damage and its relation to ictal physiological events. Arch Neurol 1973;28:10-17. 